\title{
The initial phase of oxidative stress in a steroid-induced osteonecrosis rabbit model
}

\author{
Toru Ichiseki $^{{ }^{*}}$, Ayumi Kaneuji ${ }^{1}$, Yoshimichi Ueda ${ }^{2}$, Seiji Kaneko ${ }^{1}$, Syusuke Ueda ${ }^{1}$, \\ Tadami Matsumoto ${ }^{1}$ \\ ${ }^{1}$ Department of Orthopaedic Surgery, Kanazawa Medical University, Ishikawa, Japan \\ ${ }^{2}$ Department of Pathophysiological and Experimental Pathology, Kanazawa Medical University, Ishikawa, Japan \\ Email: "tsy-ichi@kanazawa-med.ac.jp
}

Received 8 August 2012; revised 11 September 2012; accepted 19 October 2012

\begin{abstract}
Using a rabbit model, we examined $\mathbf{N}$ epsilon-(hexanoyl) lysine (HEL) levels in bone and urine to detect when peroxidative reaction first occurs after steroid administration. Japanese white rabbits weighing about $3.5 \mathrm{~kg}$ each were injected with a single intramuscular dose of methylprednisolone $40 \mathrm{mg} / \mathrm{kg}$ and divided into groups consisting of 10 rabbits each, which were killed after 1, 3, 5 and 14 days (groups $A$, $B, C$ and $D$ respectively). As a control, 10 untreated rabbits (group N) were also studied. The proximal femurs were examined histopathologically and immunohistochemically using monoclonal antibody HEL, which is a highly specific antibody against $\mathbf{N}$ epsilon-(hexanoyl) lysine, an early peroxidation marker. In addition, urinary levels of HEL were measured by enzyme-linked immunosorbent assay in group N, A, B and C. Osteonecrosis was detected only in group $D(90 \%)$. Increase of positive reaction of HEL in the bone was observed in group $A$ and $D$. HEL expression in group $D$ was judged to be a secondary reaction resulting from the development of osteonecrosis. Urinary level of HEL showed a significant increase in only group $A(P<0.001)$. The present findings suggest that peroxidation in bone occurred within 24 hours after steroid administration in a rabbit model and that it is possible to noninvasively grasp the timing of this peroxidative reaction by measuring the urinary level of HEL.
\end{abstract}

Keywords: Steroid; Osteonecrosis; Oxidative Stress; Hexanoyl Lysine

\section{INTRODUCTION}

Excessive oxidative stress-induced cell damage plays a significant role in the course of various diseases [1,2]. It

\footnotetext{
"Corresponding author.
}

has been reported that oxidative stress generated after steroid administration is also an important cause of steroid-induced osteonecrosis [3,4]. Immunohistochemical investigations have been performed also for osteonecrosis models using advanced glycation end-products (AGEs), Malondialdehyde (MDA) and 4-hydroxy-2-nonenal (4HNE) among others to determine oxidative stress in tissue. Although many reports have insisted on the involvement of each of these [3,5], none of them is capable of detecting peroxidation at its early stage since they are end products of the peroxidation reaction. In an investigation of the early stage in the bone of a rabbit model, it was demonstrated that DNA oxidation injury and ischemic event were induced at three to five days after steroid administration [4]. Accordingly, steroidinduced peroxidation in bone may occur at an earlier stage than these events.

We consider that pinpointing the initial timing of peroxidation may help to narrow the focus to clarify the mechanism of steroid-induced osteonecrosis.

Recently, a monoclonal antibody, which is capable of immunohistochemical investigation of $\mathrm{N}$ epsilon-(hexanoyl) lysine (HEL), has been developed as a new peroxidation marker of tissue. HEL is a stable initial product derived from 13-hydroperoxy-octadecadienoic acid during the oxidation process of active oxygen species, facilitating the detection of peroxidative reaction at its early stage [6,7]. Measurement of the urinary HEL level also makes it possible to detect the peroxidative reaction at the initial stage, and an Enzyme-linked Immunosorbent Assay (ELISA) kit for this purpose has been developed [8,9].

In the present study, using a rabbit model, we examined reactions of HEL in the diaphysis of proximal femur, which is the site most susceptible to the development of steroid-induced osteonecrosis in the rabbit model, early after steroid administration as well as urinary HEL levels, and also focused on how to determine the peroxidative condition in bone caused by steroid administration and to 
detect peroxidation by urinary examination.

\section{MATERIALS AND METHODS}

Forty adult female Japanese white rabbits (mean body weight $3.5 \mathrm{~kg}$ ) were injected once with methylprednisolone at $40 \mathrm{mg} / \mathrm{kg}$ body weight into the right gluteal muscle, and 10 animals each were killed 1, 3, 5 and 14 days after steroid administration. They were designated as groups $\mathrm{A}, \mathrm{B}, \mathrm{C}$ and $\mathrm{D}$ respectively, and compared with group $\mathrm{N}$, which consisted of ten rabbits that were fed under the same conditions but not injected with steroid. Immediately after the animals had been killed the bilateral femurs were isolated and fixed in $10 \%$ formalin for 1 week, and the specimens were decalcified in $10 \%$ EDTA. The specimens were then embedded in paraffin, and cut into $4 \mathrm{~mm}$ sections.

All protocols in this study were performed in accordance with the guidelines of the Animal Research Committee of Kanazawa Medical University.

\subsection{Histopathology}

Necrosis of bone and marrow tissues was examined in haematoxylin-eosin-stained preparations by light microscopy. Osteonecrosis was judged to be present when necrosis of medullary haematopoietic cells or fat cells or empty lacunae or condensed nuclei in osteocytes were noted. Osteonecrosis was judged to be present when osteonecrosis was identified in either isolated femur [10]. The rate of development of osteonecrosis was calculated as the ratio of the number of rabbits with osteonecrosis to the total number of rabbits used.

\subsection{Immunohistochemistry}

To determine the initial timing of oxidative stress in bone, the femur was stained immunohistochemically with antiHEL monoclonal antibody (Japan Institute for the Control of Aging, Shizuoka, Japan). Briefly, after deparaffinization, the sections were treated with $0.3 \% \mathrm{H}_{2} \mathrm{O}_{2}$ in methanol for $30 \mathrm{~min}$ at room temperature, and with $0.1 \%$ trypsin for $15 \mathrm{~min}$ at $37^{\circ}$. Then the sections were reacted with HEL monoclonal antibody (1:100) for $4^{\circ}$ overnight, followed by incubation with Dako EnVision/HRP system (Dako, Tokyo, Japan) for $30 \mathrm{~min}$ at room temperature. Sections were then treated with DAB for $5 \mathrm{~min}$, and counterstained with haematoxylin-eosin for $1 \mathrm{~min}$.

\subsection{Quantification of Immunohistological Data (HEL \%PA)}

Immunohistological data were quantified using Scion Image freeware and calculated as the positive area (\%PA) per visual field [4]. Briefly, five appropriate locations, which were focused on the proximal femur, were taken of each specimen at a magnification of $40 \times 5$. The brightness and contrast of each image file were uniformly enhanced with Adobe Photoshop version 7.0J, followed by image analysis with Scion Image freeware. Oxidative stress was evaluated by calculating the percentage occupied by positive cells relative to the total area in five randomly selected visual fields. Specimens from the five groups (groups N, A, B, C and D) were analysed. The mean of the data obtained from five independent fields was used as a representative value for each animal.

\subsection{Measurement of Urinary HEL Level by Enzyme-Linked Immunosorbent Assay (ELISA)}

We weighed differences between groups N, A, B and C to detect urinary HEL levels at the time of peroxidation before the development of osteonecrosis. Urinary HEL levels were determined with a competitive ELISA kit (Japan Institute for the Control of Aging, Shizuoka, Japan). In this kit, HEL concentrations were measured at an absorbance of $450 \mathrm{~nm}$. The levels of creatine in urine samples were simultaneously measured for compensation of urinary levels of HEL.

\subsection{Statistical Analysis}

Urinary HEL levels were expressed as the mean \pm SD. Statistical analysis was performed using analysis of variance with Dunnett's multiple comparison test. By using this test we were able to determine the point in time after administration of steroid that HEL \%PA and urinary HEL levels showed a significant change, as compared with the values in $\mathrm{N}$ group. $\mathrm{P}$ values less than 0.05 were considered significant. Statistical analysis was performed using StatView J-5.0 software (SAS Institute).

\section{RESULTS}

\subsection{Histopathology}

There was no necrosis of the bone or bone marrow in groups $\mathrm{N}, \mathrm{A}, \mathrm{B}$ or $\mathrm{C}$, in contrast to group $\mathrm{D}$ in which osteonecrosis was observed in 9 of 10 rabbits (90\%) (Figures 1(a) and (b)). Necrotic areas in group D were clearly demarcated from the surrounding normal tissue. Empty lacunae were found in bone trabeculae, and the surrounding bone marrow tissue also showed necrotic changes (hematopoietic cell and fat cell necrosis).

\subsection{Immunohistochemical Study}

Little reaction of HEL was observed in group N (Figure 1(c)). The intense expression at the proximal part of femur in group A made it possible to confirm that peroxidation had already occurred in the bone by 24 hours after 

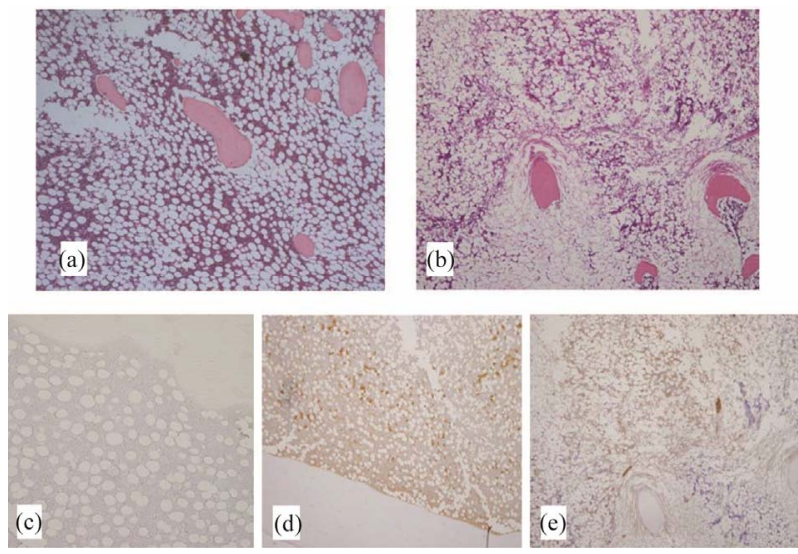

Figure 1. Histopathological and immunohistochemical studies of femurs of rabbits treated with steroid. (a) Group A (haematoxylin-eosin staining): Normal bone tissue is present with no findings of osteonecrosis; (b) Group D (haematoxylin-eosin staining): Osteocytes in the stained bone contain achromatic nuclei or empty lacunae, showing typical features of osteonecrosis. Medullary hematopoietic cells around the site of osteonecrosis are mixed with necrotic and degenerated cells, and necrotic fat cells are empty, having lost their cellular structures; (c) Group N (HEL staining): Few positive findings are observed; (d) Group A (HEL staining): Significantly intense positive reaction is observed; (e) Group D (HEL staining): Positive findings are observed at proximal part of osteonecrosis, but almost none in other areas.

steroid administration (Figure 1(d)). The reaction of HEL gradually weakened in groups B and C, and accumulation was observed just at the proximal part of the osteonecrosis in group D (Figure 1(e)). Little expression of HEL was observed in rabbits in which no osteonecrosis was detected in group D. HEL \%PA was $1.2 \% \pm$ $0.4 \%$ in group $\mathrm{N}, 10.6 \% \pm 4.7 \%$ in group $\mathrm{A}, 2.8 \pm 0.6$ in group $B, 1.8 \pm 0.5$ in group $C$ and $11.8 \% \pm 6.4 \%$ in group D representing a significant increase in groups $\mathrm{A}$ and D compared with group $\mathrm{N}(\mathrm{P}<0.001)$ (Figure 2).

\subsection{Urinary Levels of HEL}

The values of HEL were $120 \pm 28.6 \mathrm{pmol} / \mathrm{mg} \mathrm{Cr}$ in group $\mathrm{N}, 760 \pm 78.1 \mathrm{pmol} / \mathrm{mg} \mathrm{Cr}$ in group $\mathrm{A}, 211 \pm 31.2$ $\mathrm{pmol} / \mathrm{mg} \mathrm{Cr}$ in group B and $191 \pm 42.6 \mathrm{pmol} / \mathrm{mg} \mathrm{Cr}$ in group $\mathrm{C}$ respectively, representing a significantly high value only in group $A(p<0.001)$ (Figure 3 ).

\section{DISCUSSION}

In a previous study in which steroids were administered in a rabbit model, the presence of DNA oxidation injury was confirmed in the early period after steroid administration[4]. Moreover, the development of osteonecrosis has been described in rats administered the pro-oxidant buthionine sulphoximine (BSO) [11,12]. Recently, the efficacy of lipid-lowering agents [13], reduced glutathione [3] and vitamin E [14] in the prevention of ster-

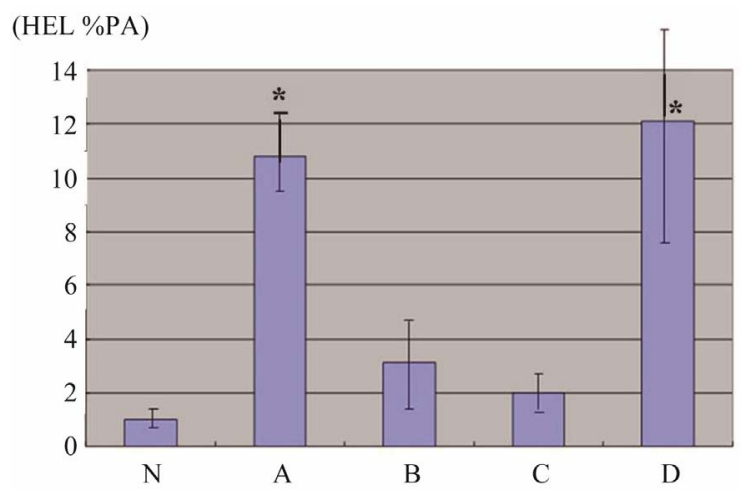

Figure 2. Quantification of immunohistological data (HEL \%PA). Significant increase is observed in groups A and $\mathrm{D}$, showing bimodal variation. Results are shown as mean \pm SD. ${ }^{*} \mathrm{P}<0.001$ vs $\mathrm{N}$ group.

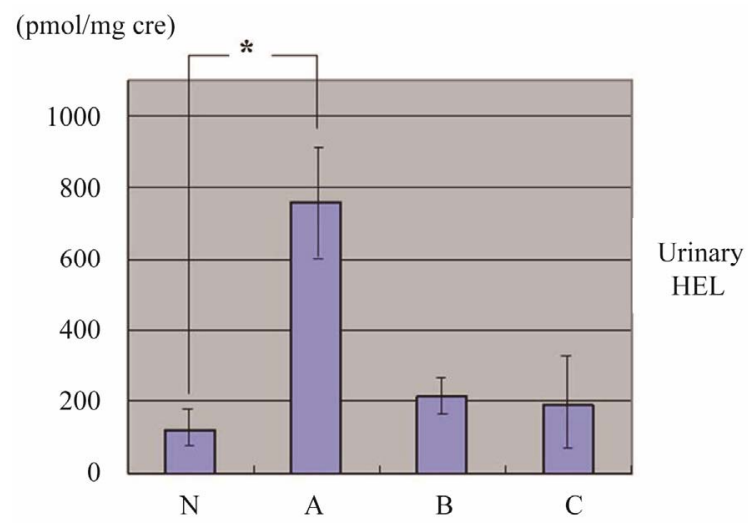

Figure 3. Urinary HEL level by ELISA. Significant increase is observed only in group A. Results are shown as mean \pm SD. ${ }^{*} \mathrm{P}<0.001$ vs $\mathrm{N}$ group.

oid-induced osteonecrosis has been reported in various animal models. These agents all exert an antioxidant effect. These findings implicate oxidative stress in the development of osteonecrosis, and suggest that by inhibiting it the incidence of osteonecrosis may be considerably decreased. Therefore, detecting the initial timing of peroxidation may help to narrow the focus to help clarify the mechanism of steroid-induced osteonecrosis as well as to devise novel prophylactic interventions.

Recently, a series of reports have shown the usefulness of HEL, which immunohistochemically can detect a tissue's peroxidative reaction in the initial stage. In such circumstances, using ELISA technique has made it possible to examine HEL levels in urine [8,9].

In this study, intense accumulation of HEL expression in the bone had already been observed at the proximal portion of femur, which is a site of predilection for steroid-induced osteonecrosis in a rabbit model, 24 hours after steroid administration, and subsequently it gradually decreased from after three days to five days. In contrast, in group $\mathrm{D}$, the accumulation was observed just at 
the proximal part of osteonecrosis and rarely observed in rabbits in which no osteonecrosis had been detected. HEL expression in group D was judged to be a secondary reaction resulting from the development of osteonecrosis, indicating that peroxidation at the initial stage sufficient to cause osteonecrosis had occurred within 24 hours after steroid administration. These findings suggest that antioxidant agents should be administered sufficiently early after steroid administration to exert an adequate effect within 24 hours so as to prevent steroid-induced osteonecrosis in this model.

On the other hand, the reason why we measured urinary levels of HEL in this study was to figure out a way of examination capable of noninvasively detecting the initial timing and intensity of peroxidation occurrence in the bone to some extent. In this study, we examined urinary levels of HEL in groups $\mathrm{N}, \mathrm{A}, \mathrm{B}$ and $\mathrm{C}$ respectively to detect oxidative changes at their initial stages after steroid administration when osteonecrosis had not yet occurred. HEL in urine and bone both underwent an extremely similar transition in which HEL showed a significant increase within 24 hours after steroid administration and decrease from after three days to five days. This suggests that measurement of urinary HEL levels may provide a clue to the state of peroxidation in bone soon after steroid administration.

From the viewpoint of prevention of steroid-induced osteonecrosis, it is necessary to detect oxidative reaction in the bone at its initial stage. However, in our daily practice, it is impossible to perform bone biopsy on a frequent basis. This study suggests that urine testing for HEL, which can be performed noninvasively in daily clinical practice, will enable clinicians to use antioxidative agents before the development of steroid-induced osteonecrosis.

\section{CONCLUSION}

The present results demonstrated that peroxidative reaction had already occurred in the bone within 24 hours after steroid administration and that the reaction could be detected by measuring the urinary level of HEL.

\section{ACKNOWLEDGMENTS}

This work was partly supported by grants 21591963 (to TI) from the Japanese Ministry of Education, Culture, Sports, Science and Technology.

\section{REFERENCES}

[1] Baynes, J.W. (1991) Role of oxidative stress in development of complications in diabetes. Diabetes, 40, 405412. doi:10.2337/diabetes.40.4.405

[2] Stocker, R. and Keaney Jr., J.F. (2004) Role of oxidative modifications in atherosclerosis. Physiological Reviews, 84, 1381-1478. doi:10.1152/physrev.00047.2003

[3] Ichiseki, T., Matsumoto, T., Nishino, M., Kaneuji, A. and Katsuda, S. (2004) Oxidative stress and vascular permeability in steroid-induced osteonecrosis model. Journal of Orthopaedic Science, 9, 509-515. doi:10.1007/s00776-004-0816-1

[4] Ichiseki, T., Kaneuji, A., Katsuda, S., Ueda, Y., Sugimori, T. and Matsumoto, T. (2005) DNA oxidation injury in bone early after steroid administration is involved in the pathogenesis of steroid-induced osteonecrosis. Rheumatology (Oxford), 44, 456-460. doi:10.1093/rheumatology/keh518

[5] Murata, M., Kumagai, K., Miyata, N., Osaki, M. and Shindo, H. (2007) Osteonecrosis in stroke-prone spontaneously hypertensive rats: Effect of glucocorticoid. Journal of Orthopaedic Science, 12, 289-295. doi:10.1007/s00776-007-1129-y

[6] Kato, Y., Mori, Y., Makino, Y., Morimitsu, Y., Hiroi, S., Ishikawa, T. and Osawa T. (1999) Formation of $\mathrm{N} \varepsilon$ (hexanonyl) lysine in protein exposed to lipid hydro-peroxide. The Journal of Biological Chemistry, 274, 2040620414. doi:10.1074/jbc.274.29.20406

[7] Kato, Y., Miyake, Y., Yamamoto, K., Shimomura, Y., Ochi, H., Mori, Y. and Osawa, T. (2000) Preparation of a monoclonal antibody to N(epsilon)-(Hexanonyl)lysine: Application to the evaluation of protective effects of flavonoid supplementation against exercise-induced oxidative stress in rat skeletal muscle. Biochemical and Biophysical Research Communications, 274, 389-393. doi:10.1006/bbrc.2000.3150

[8] Tokuda, F., Sando, Y., Matsui, H. and Yokoyama, T. (2009) N epsilon-(hexanoyl) lysine, a new oxidative stress marker, is increased in metabolic syndrome, but not in obstructive sleep apnea. The American Journal of the Medical Sciences, 338, 127-133. doi:10.1097/MAJ.0b013e3181a478e5

[9] Ueno, Y., Horio, F., Uchida, K., Naito, M., Nomura, H., Kato, Y., Tsuda, T., Toyokuni, S. and Osawa, T. (2002) Increase in oxidative stress in kidneys of diabetic Akita mice. Bioscience, Biotechnology, and Biochemistry, 66, 869-872. doi:10.1271/bbb.66.869

[10] Yamamoto, T., Irisa, T., Sugioka, Y. and Sueishi, K. (1997) Effects of pulse methylprednisolone on bone and marrow tissues: Corticosteroid-induced osteonecrosis in rabbits. Arthritis \& Rheumatism, 40, 2055. doi:10.1002/art.1780401119

[11] Ichiseki, T., Ueda, Y., Katsuda, S., Kitamura, K., Kaneuji, A. and Matsumoto, T. (2006) Oxidative stress by glutathione depletion induces osteonecrosis in rats. Rheumatology (Oxford), 45, 287-290. doi:10.1093/rheumatology/kei149

[12] Ichiseki, T., Kaneuji, A., Ueda, Y., Nakagawa, S., Mikami, T., Fukui, K. and Matsumoto, T. (2011) Osteonecrosis development in a novel rat model characterized by a single application of oxidative stress. Arthritis \& Rheumatism, 63, 2138-2141. doi:10.1002/art.30365

[13] Nishida, K., Yamamoto, T., Motomura, G., Jingushi, S. and Iwamoto, Y. (2008) Pitavastatin may reduce risk of 
steroid-induced osteonecrosis in rabbits: A preliminary histological study. Clinical Orthopaedics and Related Research, 466, 1054-1058. doi:10.1007/s11999-008-0189-4

[14] Mikami, T., Ichiseki, T., Kaneuji, A., Ueda, Y., Sugimori,
T., Fukui, K. and Matsumoto, T. (2010) Prevention of steroid-induced osteonecrosis by intravenous administration of vitamin E in a rabbit model. Journal of Orthopaedic Science, 15, 674-677.

doi:10.1007/s00776-010-1516-7 